\title{
Clemens Fuest*
}

\section{Christian Watrin - Ökonom, Hochschul- lehrer, Politikberater}

https://doi.org/10.1515/zfwp-2021-2051

Christian Watrin gehört zu den deutschen Ökonomen, die wirtschaftspolitische Debatten der siebziger und achtziger Jahre ebenso geprägt haben wie die Diskussionen über die deutsche Wiedervereinigung und die europäische Integration nach dem Fall des Eisernen Vorhangs im Jahr 1989. Er wird als Schüler und Mitarbeiter von Alfred Müller-Armack zu den Vertretern der ordoliberalen wirtschaftspolitischen Schule gezählt. Durch die Breite seiner wissenschaftlichen Arbeit und seiner Tätigkeit als Politikberater entzieht er sich aber der Einordnung in Schubladen.

\section{Ökonomische Probleme von Marktzugangs- schranken}

Als junger Wissenschaftler hat Christian Watrin bereits mit seiner im Jahr 1957 vorgelegten Dissertation auf sich aufmerksam gemacht. Sie hat den Titel „Der Befähigungsnachweis in Handwerk und Einzelhandel“ und befasst sich mit der bis heute aktuellen und umstrittenen Frage, ob es sinnvoll und gerechtfertigt ist, von Handwerkern einen Meisterbrief zu verlangen, wenn sie ein Handwerksunternehmen gründen wollen. Diese Vorschrift hat eine lange Tradition, die bis zu den mittelalterlichen Zünften zurückreicht. Das einleitende Kapitel der Dissertation gibt einen bis heute höchst lesenswerten und spannenden Überblick über die wechselvolle Geschichte des Befähigungsnachweises und verdeutlicht, wie Marktzugangsbeschränkungen sich im Spannungsfeld zwischen staatlichen Steuerungsvorstellungen, Partikularinteressen und liberalen Forderungen nach Gewerbefreiheit entwickelt haben.

Durch die Stein-Hardenbergschen Reformen der Jahre 1810/1811 wurden die noch bestehenden Zunftregeln in Preußen zu Gunsten einer freiheitlichen Gewerbeverfassung abgeschafft. Die etablierten Handwerker liefen Sturm gegen diese Liberalisierung und erreichten, dass sie größtenteils wieder aufgehoben wurden.

*Kontakt: Clemens Fuest, Ludwig-Maximilians-Universität München und ifo Institut, E-Mail: fuest@ifo.de. 
In den folgenden Jahrzehnten wechselten sich Liberalisierungen und neuerliche Zugangsbeschränken immer wieder ab. Nach dem zweiten Weltkrieg gaben die amerikanischen Besatzer vor, dass Gewerbefreiheit gelten sollte, wenn nicht Gründe des Gesundheitsschutzes, der öffentlichen Sicherheit oder andere Gemeinwohlgründe Beschränkungen verlangten. Kaum hatte die junge Bundesrepublik die Gewerbepolitik wieder übernommen, begannen die Gewerbeverbände, sich für eine neuerliche Beschränkung der Gewerbefreiheit einzusetzen. Trotz aller Bekenntnisse zu einer liberalen marktwirtschaftlichen Ordnung wurde 1953 für alle Handwerkszweige der große Befähigungsnachweis wieder eingeführt.

Christian Watrin analysiert in seiner Arbeit akribisch die verschiedenen ökonomischen Gründe für und wider den Befähigungsnachweis. Dabei werden Fragen untersucht, die in der modernen Wirtschaftstheorie eine zentrale Rolle spielen, beispielsweise Informationsasymmetrien zwischen Produzenten und Konsumenten. Was die Rechtfertigung des Befähigungsnachweises als Marktzugangsbeschränkung mit Marktunvollkommenheiten angeht, kommt der Autor zu einem klaren Urteil: „Die vorliegende Untersuchung wollte deutlich machen, dass die Reaktivierung eines aus der Zunftzeit überkommenen gewerbepolitischen Mittels in der Gegenwart vielfältige Reibungen und Störungen im Wirtschaftsprozess verursacht.“1

\section{Außenwirtschaftstheorie und internationale Handelskonflikte}

Während er seine Dissertation bei dem Kölner Wirtschaftsprofessor Theodor Wessels schrieb, war er damals bereits mit Alfred Müller-Armack in Kontakt und hat in den folgenden Jahren eng mit ihm zusammengearbeitet. Seine Habilitationsschrift wendet sich Fragen der internationalen Wirtschaft zu und hat den Titel „Weltwirtschaft und Außenhandel: Zur Theorie ökonomischer Konflikte“. Auch dieses Thema hat bis heute nicht an Aktualität verloren. Handelskonflikte sind vor allem durch die protektionistische Orientierung des US-Präsidenten Donald Trump und die Spannungen zwischen den USA und China gerade in den letzten Jahren in den Mittelpunkt wirtschaftspolitischer Debatten getreten.

In der Habilitationsschrift und weiteren Publikationen der frühen sechziger Jahre stehen zwischenstaatliche Konflikte in Außenhandelsfragen im Fokus. Dabei werden vor allem die sehr verschiedenen Perspektiven der merkantilisti-

1 Watrin (1958), S. 266. 
schen Außenhandelstheorie einerseits und der Theorie der komparativen Kostenvorteile andererseits untersucht. Während der Merkantilismus Außenhandel als Konflikt betrachtet, bei dem es Gewinner und Verlierer gibt, betont die Theorie der komparativen Kostenvorteile die beidseitigen Gewinne, die durch den Außenhandel entstehen. Die kooperative Sichtweise des Außenhandels hat Christian Watrins Auffassungen zum Außenhandel geprägt, allerdings hat er sich in späteren Jahren intensiv mit Weiterentwicklungen der Außenhandelstheorien, vor allem Theorien des intraindustriellen Handels und den damit verbundenen wirtschaftspolitischen Implikationen, beschäftigt.

Nach Abschluss seiner Habilitation wurde Christian Watrin zunächst auf eine Professur an der Ruhr-Universität Bochum berufen. Im Jahr 1971 wurde er Nachfolger Alfred Müller-Armacks als Inhaber des Lehrstuhls für Wirtschaftliche Staatswissenschaften am Wirtschaftspolitischen Seminar der Universität zu Köln und Direktor des angegliederten Instituts für Wirtschaftspolitik.

\section{Gesellschaftspolitische Kontroversen und Kapitalismuskritik der siebziger Jahre}

Christian Watrins Arbeiten aus den siebziger und frühen achtziger Jahren spiegeln die intensiven damaligen Debatten über die Entwicklung der marktwirtschaftlichen Ordnung, die wieder aufkommende und sich verstärkende Kapitalismuskritik und die Hinwendung zu marxistischen Gesellschaftsanalysen sowie Ideen über alternative Wirtschaftssysteme wider. Charakteristisch für die Arbeiten aus dieser Zeit ist, dass die Argumente der Kritiker der marktwirtschaftlichen Ordnung in großer Klarheit herausgearbeitet und dann an empirischen Entwicklungen gemessen werden. Ein Beispiel dafür ist der 1976 in der Fachzeitschrift Wirtschaftspolitische Chronik erschienene Aufsatz „Neuer Klassenkampf: Bemerkungen zum Erklärungswert der Klassenkampflehren“. Dort kontrastiert Christian Watrin die marxistische These der zunehmenden Konzentration und Polarisierung wirtschaftlicher Macht mit der empirischen Beobachtung eines wachsenden unternehmerischen Mittelstands und folgert daraus: „Gibt man ... wie die neue Linke, den Bezug auf die ökonomische Basis, die Lehre von der Zerstörung der ökonomischen Mittelstände durch die großbetriebliche Konkurrenz preis, so gibt man damit auch den Anspruch der Marxschen Klassenkampflehre auf, die Realität zu erklären.“2

2 Watrin (1976), S. 36. 
Andere Beiträge aus dieser Zeit beschäftigen sich mit Fragen der sozialen Gerechtigkeit, der Messung ökonomischer Effizienz oder der politischen Ökonomie der Inflation. Eine Konstante, die sich durch dieses Werk zieht, ist das besondere Interesse am Verhältnis zwischen individueller Freiheit, der Rolle des Staates und der Forderung nach Gleichheit. Christian Watrin war dabei deutlich beeinflusst von Ideen des Ökonomie-Nobelpreisträgers Friedrich August von Hayek, den er in seiner Zeit als Privatdozent persönlich kennengelernt hatte, während einer Lehrstuhlvertretung an der Universität Freiburg. Charakteristisch für diese Arbeiten ist der in der Zeitschrift ORDO im Jahr 1979 publizierte Aufsatz „Grenzen der Gleichheit in einer freiheitlichen Ordnung“, der das Spannungsverhältnis, aber auch Komplementaritäten zwischen Freiheit und Gleichheit ausleuchtet. Christian Watrin hat in diesen Jahren Beiträge zur Entwicklung des Konzepts der Sozialen Marktwirtschaft als Wirtschafts- und Gesellschaftsordnung geleistet, die bis heute wegweisend sind.

Angesichts der intensiven Beteiligung an wirtschafts- und gesellschaftspolitischen Debatten in Deutschland sollte nicht übersehen werden, dass Christian Watrin mehr als viele Ökonomen seiner Zeit in die internationale Wirtschaftsforschung eingebunden war und enge Kontakte vor allem in die USA und ins Vereinigte Königreich unterhielt. Im akademischen Jahr 1986-1987 war er Inhaber des Konrad Adenauer Lehrstuhls an der Georgetown University in Washington D.C. Er hat sich intensiv mit den wissenschaftlichen Arbeiten von James Buchanan und Geoffrey Brennan beschäftigt und war Herausgeber der deutschen Übersetzungen ihrer Bücher. Von 2000 bis 2002 war er Präsident der Mont Pelerin Society, einer internationalen Vereinigung liberaler Ökonomen. Seine Vorgänger in diesem Amt waren neben dem Gründungspräsidenten Friedrich A. von Hayek Ökonomen wie Milton Friedman, Gary Becker, Herbert Giersch und George Stigler.

\section{Europäische Währungsunion und die Wieder- vereinigung Deutschlands}

In den achtziger Jahren intensivierte sich die Debatte über die monetäre Integration in Europa und das Projekt einer europäischen Währungsunion. Christian Watrin war zutiefst überzeugter Anhänger der europäischen Integration. Gleichzeitig sah er mit Sorge, dass angesichts der vorliegenden Planungen für eine europäische Währungsunion ein Ungleichgewicht drohte zwischen der auf nationaler Ebene verbleibenden Verantwortung für die Finanz- und Wirtschaftspolitik und der Europäisierung der Geldpolitik. Er war Mitunterzeichner eines viel beachteten Ökonomenaufrufs aus dem Jahr 1992 mit dem Titel: „Die währungspolitischen Beschlüsse 
von Maastricht: Eine Gefahr für Europa“. In dem Text wird davor gewarnt, dass die Währungsunion durch Probleme der Wettbewerbsfähigkeit ökonomisch schwächerer Mitgliedstaaten in Schieflage geraten würde, umfangreiche Transfers erforderlich werden könnten, um die Währungsunion aufrechtzuerhalten, und letztlich die Preisniveaustabilität gefährdet sein könnte. ${ }^{3}$ Die Inflationsraten im Euroraum waren trotz zwischenzeitlicher Krisen niedrig, aber die Sorge, dass es zu Ungleichgewichten und zwischenstaatlichen Transfers kommt, hat sich bestätigt.

Das wichtigste ökonomische und politische Ereignis der 1980er Jahre war aber nicht der Beginn des Projekts der Europäischen Währungsunion. Es war der Fall des Eisernen Vorhangs, und mit ihm der Beginn des Prozesses, der letztlich zur deutschen Wiedervereinigung führte. Christian Watrin hatte Zentralverwaltungswirtschaften immer für hochgradig ineffizient gehalten, aber ihr Kollaps und damit verbunden das Ende der kommunistischen politischen Systeme in Europa im Jahr 1989 kam auch für ihn überraschend, wie er selbst betont hat. Die Schwierigkeit, derartige Umwälzungen vorherzusehen, hat er in einer Rede vor der Philadelphia Society im Jahr 1999 so beschrieben:

„As a rule political economists are not very good at forecasting the longrun stability of complex institutions. Otherwise, they should have been able to predict the collapse of the Soviet empire - ending Socialism in its heartland and in satellite countries - in time. In explaining this oversight two arguments could be mentioned: First, the Hayekian one that the future is not only unknown, but to a certain degree unknowable, and second, that our knowledge about the functioning of institutions and, especially, the conditions under which a break-down can occur, is still rather limited." ${ }^{4}$

Diese Analyse und die dabei durchscheinende Skepsis bezüglich der Möglichkeiten, ökonomische und historische Entwicklungen vorherzusagen, verbunden mit Distanz zum Untersuchungsthema, ist typisch für Christian Watrins Argumentationsweise. In den Jahren nach 1989 haben die Transformation der ehemaligen Zentralverwaltungswirtschaften und die deutsche Wiedervereinigung ihn sowohl in seinen wissenschaftlichen Arbeiten als auch in der Politikberatung intensiv beschäftigt. Die Debatte über die Entscheidung zwischen der Rückerstattung von enteignetem Vermögen und der Entschädigung der zwischenzeitlichen Eigentümer, die deutsch-deutsche Währungsunion, die sprunghaft ansteigende Arbeitslosigkeit, die Privatisierung und Abwicklung ostdeutscher Unternehmen durch die Treuhand gehörten zu den drängenden Themen dieser Zeit.

3 Eine Zusammenfassung seiner Analyse zu Lage, Problemen und Perspektiven der EU und der Währungsunion findet sich in Watrin (1999).

4 Watrin (1999). 


\section{Christian Watrin und die Freiheit des universitären Lebens}

Von 1991 bis 1995 war ich als Wissenschaftlicher Mitarbeiter und Doktorand an seinem Lehrstuhl beschäftigt. Christian Watrin war damals ein weit über die Grenzen seines Faches bekannter Wissenschaftler und gefragter Politikberater und Kommentator in den Medien. Er hatte Rufe an Universitäten im In- und Ausland erhalten, unter anderem an die Universität Wien. Er war zwischenzeitlich Vorsitzender des Wissenschaftlichen Beirats beim Bundeswirtschaftsministerium und hochdekoriert, unter anderem mit dem Bundesverdienstkreuz. Ich hatte als sein Mitarbeiter aber nie den Eindruck, dass all diese Ämter und Ehrungen ihn sonderlich beeindruckt haben. Sein primäres Interesse galt der wissenschaftlichen Arbeit, der Beratung von Politik und Öffentlichkeit und der Frage, welche Implikationen wissenschaftliche Erkenntnisse für wirtschaftspolitisches Handeln haben. Ich erinnere mich an zahlreiche, oft bis tief in die Abendstunden reichende Diskussionen und Vorträge, meistens in den Lehrstuhlräumen im 7. Stock des Wiso-Hochhauses der Universität zu Köln. Christian Watrin war in diesen Debatten sehr engagiert, aber niemals dogmatisch. Er hatte große Freude daran, Argumente zu entwickeln und anderen zuzuhören, auch Nachwuchswissenschaftlern und Studierenden, und kontroverse Diskussionen zu führen. Seine Perspektive war immer kosmopolitisch und menschenfreundlich. Er liebte die geistige Freiheit und die freie Rede. Sie waren nach seiner Vorstellung der wahre Kern dessen, was universitäres Leben bedeutet. Darin war und ist er für mich und viele Kolleginnen und Kollegen Vorbild.

Im Dezember des vergangenen Jahres verstarb Christian Watrin im Alter von 90 Jahren in seiner Heimatstadt Köln. Seine Beiträge zur ökonomischen Forschung und Lehre und zur wirtschaftspolitischen Beratung werden Bestand haben.

\section{Literatur}

Watrin, Christian (1958), Der Befähigungsnachweis in Handwerk und Einzelhandel, Dissertation, Universität zu Köln.

Watrin Christian (1976), „Neuer Klassenkampf: Bemerkungen zum Erklärungswert der Klassenkampflehren“, Wirtschaftspolitische Chronik Jg 25, S. 23-36.

Watrin, Christian (1979), „Grenzen der Gleichheit in einer freiheitlichen Ordnung“, ORDO Bd. 30, S. 159-175.

Watrin, Christian (1999), "Will the European Union Survive the 21st Century?"

Paper Presented to The Philadelphia Society, April 24,1999, https://phillysoc.org/watrin-willthe-european-union-survive-the-21st-century/. 